\title{
Supporting the Development of Transparent Interaction
}

\author{
Jakob E. Bardram and Olav W. Bertelsen \\ Computer Science Dept., Aarhus University \\ Ny Munkegade 116, DK-8000 Aarhus C. Denmark \\ bardram@daimi.aau.dk, olavb@daimi.aau.dk
}

\begin{abstract}
Transparency has been seen as a significant aspect of successful humancomputer interaction. In this paper we investigate this concept from the point of view of activity theory. We show that transparency cannot be understood as a static feature of the interface, but that the crucial point in achieving transparent interaction is the ongoing development of unconscious operations, embedded in the process of use. We suggest that the process of deliberate formation and refinement of operations during the course of interaction, is supported by setting conditions for the creation of a zone of proximal development in the interface.
\end{abstract}

\section{Introduction}

Better interfaces is a goal shared by most people in the HCI community, but it is harder to agree on what constitutes a good interface; and to what extent a scientific foundation for design is necessary. In the visionary contribution to a scientific foundation of HCI by Card, Moran, \& Newell (1983; Newell \& Card 1985), the criteria for good interaction were mostly of quantitative nature; maximum speed, minimum errors, fast learning, and other measurable quantities. The degree to which such criteria are met is easily assessed in an objective manner; unfortunately, many important usability aspects are not covered by such metrics.

A recent report sponsored by the US National Science Foundation claims that "The aim of HCI research should be to understand the principles behind what is necessary for transparent interaction." (Strong 1994, p.19), thus suggesting that transparency could be a measure for good interface design. It has been recognised by many in the HCI community (e.g. Hutchins et al. 1986; Laurel 1986, Winograd \& Flores 1986; Shneiderman 1992) that people want to think about the job they are doing and not about the computer artefact they are using; but it is hard to find substantial explanations of the concept of transparency.

In specific situations it may be easy to see whether a computer artefact appears to be transparent to the user. Experienced Emacs users interact transparently with the editor although it appeared completely opaque at their first encounter with it. Emacs is a very powerful and transparent tool for many programmers, but it will probably be hard to persuade writers to use it. On the other hand, it is not likely that an artefact like MS-Word that many writers interact transparently with, can become a transparent tool for programming. This small example illustrates two things about transparency. Firstly, that transparent interaction is not a property of the interface by itself, but a quality of the use activity; and secondly, that transparent interaction is developed by the user during interaction. 
In this paper we discuss the concept of transparency from the point of view of activity theory, and we try to identify how transparency is developed during use, and how this ongoing process can be supported by conditions in the interface.

\section{HCI and the information processing paradigm}

From the very beginning two distinct tendencies have coexisted in the field of HCI. One side mainly motivated by a practically oriented concern for the usability of computer artefacts; the other side more oriented towards the theoretical foundation of HCI. Norman's (1981) classical analysis of UNIX can be seen as an instance of the first, Card, Moran and Newell (op. cit.) as an instance of the later. On both sides information processing is the common framework; thus, understanding the user is often referred to as, "understanding the limitations of human information processing" (Strong op. cit., p.5). The predominant tendency in the field has been pragmatic and inclusive; a book like User Centred Systems Design (Norman and Draper (eds.) 1986), is an example of this, although often referred to as cognitive science.

With respect to aspects of interaction that can be regarded stable, or constant over time, the information processing based tradition has established valuable knowledge concerning cognitive abilities of generalised users like the "expert user", ranging from studies of performance at a keystroke level (Card, Moran \& Newell, 1983) to high level concepts like direct engagement (Hutchins et. al. 1986). Due to the explicit or implicit idea of information processing, the recurrent problem is that the HCI community tends to see their task as one of fitting two separated, more or less stable engines to each other.

The most simple information processing based models like GOMS (Card et al. op cit.) are extremely additive, and any notion of context is absent; typing an " $\mathrm{H}$ " is basically the same thing no matter whether it is the first letter in "Hoovercraft" or it is pressed to get on-line help. It is impossible to explain how the same text editor can be different things in different situations - how the use qualities of computer artefacts develop in the process of use. Within such cognitive models accounts on learning are mostly of a quantitative nature, the power law of practice (Snoddy 1926; Card et al. 1983) describing how the speed of a human act develops as a function of repetition, being one of the most prominent examples.

In more elaborate models like production systems as ACT* (Anderson 1983) and SOAR (Newell 1990) the additivity is not obvious, and the models explicitly try to deal with more qualitative aspects of learning and development. The problem remains that the way human beings relate to their surroundings is understood as a kind of problem solving, or search in a problem space; and skill acquisition is thus understood in terms of chunking or other "shortcuts" in the problem-space-search.

Such models can describe how skills in operating an advanced piece of technology can become automatic. The way users relate to their surroundings is however still seen as an exchange of information between distinct entities; thus the subject-object relation is seen as secondary compared to the internal and isolated processing of information. By drawing this distinction between the users as information processing engines and their surrounding, the information processing paradigm precludes itself from understanding how the objective conditions of the world and their cognitive representations are inseparable parts of cognition. This separation is problematic in 
understanding transparency because it separates the description of development from the description of the use-activity in which development is embedded.

Although it is possible to deal with some of the dynamic aspects of the relation between users and artefacts within information processing psychology, such accounts tend to become exorbitantly complicated. We will not enter into a philosophical discussion about the general validity of different approaches to human cognition; after all, no objective or value-free methods for settling such abstract controversies exist; the choice of scientific foundation can never be legitimated by an external norm or method; we have to take the responsibility for the way we choose to understand and act in the world in which we live (Feyerabend 1975, Habermas 1972). We will, however, show that a theoretical foundation for HCI, based on the dialectical nature, and fundamental situatedness of the user-computer relation, can yield a more useful understanding of transparency than an approach dealing with the dynamic relations as an exception. By changing the basic assumptions from the mechanical materialism of cognitive science to the dialectical materialism of activity theory, it becomes possible to understand how transparency is tied to the concrete context of use, and how transparency develops within this context.

\section{The structure and development of human activity}

Human activity has three fundamental characteristics; firstly, it is directed towards a material or ideal object which distinguishes one activity from another; secondly, it is mediated by artefacts (tools, language etc.); and thirdly, it is social within a culture (Vygotsky 1978). Computer artefacts, like all other artefacts, in this way mediate human activity within a practice. By acting in the world, human beings meet the objective world, which is experienced through the activity. Thus, human knowledge about the world is reflection obtained through activity, constituting the basis for expectations, and desires about activities in this world.

Human activity can be described as a hierarchy with three levels: activities realised through chains of actions, which are carried out through operations. At each of these levels the objective world is reflected through the activity. Human activity is always directed toward a material or ideal object satisfying a need. The subject's reflection of, and expectation to, this object characterises the motive of the activity. Human activity is carried out through actions, realising objective results. These actions are controlled by the subjects conscious goals. Goals are the reflection of the objective results of the action. Actions are realised through a series of operations; each determined by the concrete physical conditions of the action. These operations are performed without thinking consciously but are oriented in the world by a nonconscious orienting basis of the operation. This orienting basis is established through experience with the concrete material conditions for the operation, and is a system of expectations about the execution of each operation controlling the operation, in the process of the activity (Leontjev 1978).

Human work is collaboration mediated by different artefacts in order to realise common objectives. The overall objective of the organisation is divided into various activities, each having its own object and performed by different persons. Each person takes part in the activity of getting the whole organisation to work. The collaborative way of doing things and the division of work between workers in the organisation form a working culture - a practice. Means of dividing work, norms, and lan- 
guage can all be seen as artefacts mediating this practice: they are made by human beings and mediate the relations between human beings and their material and social surroundings. A secretary may need to write a letter to remind Mr. Smith that he is late with his payments and is therefore motivated to initiate actions to achieve this. This activity is carried out through actions, realising objective results: to write a letter the secretary must find an address, formulate the letter, get a signature, etc. Activity and actions cannot be reduced to each other: Various actions can result in a letter handwriting, typing or using a word-processor - all different actions, mediated by different artefacts, obtaining different results but still fulfilling the same objective of writing a letter. On the other hand, the same action can be a part of realising different activities. Printing a letter on the laser-writer can both be part of the paper-archivemaintenance activity and the send-a-reminder-to-Jack-Smith activity. The analytical level of actions describes the intention of an activity - what results there are or should be obtained. Operations describe the operational level of the activity - how the action is realised, determined by the actual physical conditions of the action. The layout of the keyboard, the word processor's interface, etc. determine how the secretary uses the computer to realise the goal of writing the letter. The non-conscious orienting basis of the operation enables the skilled secretary to write the letter without directing attention to the layout of the keyboard.

Within the framework of activity theory, transparent interaction can be defined as handling the computer through operations. In this way the computer artefact mediates the activity by allowing users to perform intended actions directed on the object (inside or outside the computer) through the interface. At a given moment the user has a certain repertoire of operations; the only way interaction can be transparent is if conditions of the interface triggers operations in this repertoire (Bødker 1991).

Human activity is not stable but is transformed constantly. An activity can loose its motive and become an action with the former motive as goal. An action can loose its conscious goal and be subsumed under another action as an operation. This process of automation happens through practice. The reverse process of conceptualisation happens when the conditions for the operation force users to think consciously about what they are doing. This happens in a breakdown situation (Winograd \& Flores 1986), where the physical conditions for the operation do not match the operations orienting basis, or it happens due to deliberate focus shift from the goal of the action to the conditions for the operation (Bødker 1991). Such breakdown situations or intended focus shifts where the computer becomes object of conscious examination interrupt the transparency.

The user's repertoire of operations is constantly developing, allowing the computer artefact to become an effective support in a work situation. The operations used by skilled Emacs users were not present when they started to use the editor, they were established during use. At first the act of marking, copying and pasting some text is done by consciously taking one step at a time, deliberately making sure that the different acts obtain the desired result. The conditions of the editor for placing the cursor, marking the text, etc., are incorporated into the orienting basis. Through practice the different parts of the action become automatic and finally the total action is automated into an operation, which can be executed without conscious reflection. But if for instance the conditions for an operation change due to changing mode of the editor, the user may experience a breakdown. This conceptualisation makes the relation between the conditions and the orienting basis of the operation an object for conscious reflection, in order to fix the discrepancy. 


\section{Development of operation through automatisation of actions}

The main mechanism for the development of a repertoire of operations is automatisation of actions to operations, where the conscious guidance of the action toward a goal has stopped. This does not mean that the user does not perceive the conditions for the operation, instead the operation is oriented by an unconscious perception of these conditions.

The efficiency of the transformation of actions into operations is determined by the formation of the actions. According to Gal'perin (1969) there are three basic parameters characterising the quality of an action. Its generality, its level of abbreviation and the extent to which the action is mastered.

The generality of an action is determined by the orienting basis of the action. To generalise an action is to distinguish the cues of the objective conditions, which are necessary for the fulfilment of the action. We can differentiate various forms of orienting bases for an action, each determined by the learning process in which the action is developed. Trial and error learning results in an orienting basis solely directed towards the actual action. Such an orienting cannot be transferred to other actions and represents the lowest generality. The most general orienting basis is established through analytic learning of general methods of analysing the conditions of the action. These methods of analysis are incorporated into the orienting basis of the operation as methods for recovering from breakdown situations permitting the user to isolate the structure of the conditions for later recognition or repetition. Transparency of interaction is closely connected to the generality of the orienting basis. Abbreviation is the process of optimising the action by skipping unnecessary operations and replacing difficult operations with simpler ones, as a result of possibilities in their conditions and knowledge of their result.

When generalising an action users come to know the full scope of conditions in the surroundings that the action matches; and the full scope of potential desirable goals that the action can fulfil. When generalising the action of invoking the UNIX "cat"-command users begin to use the command not only for concatenating files but also for printing files on the screen, feeding them into pipes etc. To do this users need a general orienting basis. Similarly, abbreviation depends on the creation of the orienting basis. Removing a file from a UNIX file system may, for the "inexperienced" user, involve the execution of one or more "ls"-commands (list files in the current directory) to make sure what is going on, in addition to the "rm <filename>"-command (remove the file). When the user gets to know what is going on the remove action is abbreviated by skipping the "ls"-commands. Similarly, using a keyboard short-cut on a window based interface can be seen as an abbreviation of the more complex mouseoperated menu selection.

Finally mastering the new action is the ability to independently perform the action with new materials in unanticipated contexts, in order to obtain a new result. If users learn to drag and drop objects into the Macintosh trash can the mastering of the drag and drop action implies that users, on their own, are able to drag and drop objects into folders etc. Users do not come to master an action by watching others, but only by practising the operation themselves.

The process of establishing the action is a conscious process in which the user forms an orienting basis according to the material conditions for the action. Once the action has attained a certain level of generalisation, abbreviation, and mastery, it can 
be automated as an operation. Once the action has been automated, the process of enhancement has stopped. It is only possible to develop the operation by conceptualising it back to an action, consciously reforming this action and then automating the changed action.

When development is embedded in the daily work, one problem is that the process of refining a new action requires time. In a given work situation users may not be interested in mastering brief and general operations; the primary motive is to get the job done (quickly). In such situations learning is biased toward trial-and-error learning which establishes infertile operations only adjusted to the conditions for this specific situation.

\section{Setting the conditions for development of transparent interaction}

Mediated and social human activity builds up the human world as artefacts embodying human capabilities, developed through socio-historical practice. Human work is characterised by the collaborative production of artefacts, each made with the purpose of mediating a certain activity. This activity is therefore objectified (or crystallised) into these artefacts, and through use, the artefacts are again modified and shaped to meet the evolving human needs. Window based interfaces are artefacts, which objectify human experiences with computer interaction. An artefact like the Macintosh is a material objectification of a large number of experiences obtained during several years, ranging from the invention of the mouse by Doug Engelbart over the pioneering steps of the Xerox Star to the latest work on intelligent agents.

To assimilate the human world as made by artefact embodying human experiences, the human being must perform overt activity with the artefacts; activity adequate to the human activity embodied in these artefacts. Furthermore, this activity should be socially mediated by other people within the culture. It is a fundamental notion of activity theory that human beings, guided by other human beings, gradually assimilate the human world by reproducing the activity embodied in the artefacts. The child learns to use a spoon, not on its own, but with the help of the mother. By imitating the activity that was originally crystallised into the spoon the child tries to master it as a human artefact. Similarly, users rely heavily on colleagues for developing their skills and learning about the computer, especially novice users (Bannon 1986). Vygotsky captures this social mediation of human cognitive development in the concepts of "the zone of proximal development":

"It is the distance between the actual developmental level as determined by independent problem solving and the level of potential development as determined through problem solving under adult guidance or in collaboration with more capable peers" - Vygotsky 1978, p. 86

The concept of a zone of proximal development describes how the mediated activity develops dialectically through contradiction between what users can do with the tool today, their actual competence, and what they want to be able to do in a near future, their potential competence.

The actual competence of the user forms the basis for development; it is the user's species specific, cultural and individual experiences - what the user has learned up to this point in time. Traditionally HCI has looked at the actual competence of the general user. Direct manipulation, for instance, captures some of the hu- 
man experience as a specie and enables a "natural" interaction: human beings manipulate objects by grabbing, dragging, lifting them; the shape of objects is perceived according to the normal direction of light from the sun, etc. A more significant part of the user's actual competence is the "computer-cultural experience"; i.e. culturally established ways of interacting with computers. Window based operating systems provides a type of interaction, which is becoming gradually more culturally objectified through the common use of windows, mouse, buttons, scroll bars, pull-down menus, etc. Consistency in the use of a window based interaction makes it possible to transfer experience from one system to another. Hence, traditional HCI has searched for general principles of how computer artefacts can conform to the cultural- and species specific experiences of users. The individual experience, however, is impossible to grasp in general terms, and thus widely ignored by the field of HCI. It is, nevertheless, a fundamental part of the actual competence that has to be considered.

Based on the actual competencies the user must be able to look ahead of the actual zone of mastery and create a zone of proximal development. Gal'perin (1969) points out that children are unable to perform a task independently, if they have no complete orienting basis. Thus, learning of a new task requires that users are given an initial familiarity with the task; otherwise learning will be reduced to 'blind' trial and error. Human beings can deliberately create or seek special conditions with the purpose of learning, engaging themselves in an exploratory learning activity. According to Bol (1984), Vygotsky's concept of a zone of proximal development has a double function with respect to cognitive development. Firstly, learning activity creates a zone of proximal development and, secondly, learning is collaboration with "more capable peers". To develop transparent human-computer interaction we need to establish the right conditions for this learning activity — be it with or without "more capable peers". In this paper we use the concept of the zone of proximal development in the metaphorical sense as a zone the user is able to set, supported by the conditions built into the interface.

Three issues concerning the development of transparent interaction emerge from the general understanding of the development of human activity: Firstly, development only takes place within a purposeful use-activity. Establishing competencies as a repertoire of operations must correspond to a certain goal and motive. Without establishing this dialectic relation between learning and use, development is impossible. Secondly, the user must be able to reflect the potential mastering of the computer, and how that level of the use-activity can be reached. Learning of a new task requires an initial familiarity with it in order to "look into" the zone of proximal development - otherwise learning will be 'blind' trial and error. Finally, the user must be provided with conditions for consciously establishing actions that are general, abbreviated and mastered to a certain degree; and for automating these new action into new operations through practice. In this way, the orienting basis of the coming operation is consciously formed and optimised according to the material conditions.

These issues concern general aspects of users' surroundings that should be met to enable the development of new operations. For example: the abbreviation of mouse operations into keyboard short-cuts depends on conditions set at the interface by indicating the short-cuts next to the command names in the menu instead of placing a description of all shortcuts in a manual, forcing the user to interrupt the work-activity and go look for the manual. Similarly, the mastering of brief and general operations requires time, but this may be inhibited by conditions of the work environment such as stress, causing the user to develop infertile operations. Development of interaction 
is not isolate from "the rest of the world", and the concept of human activity emphasises this connectedness.

\section{Designing for transparency}

It is impossible to identify general "transparent features" to be included in all user interfaces and list these as general applicable guidelines for "the design of transparent computer applications". The conditions in the interface that are necessary for the development of transparent interaction depend on the users and their jobs, and the way the introduction of the new computer artefact intervenes and changes this relation. This means that in the design of computer applications we have to rely on participatory design methods like prototyping and organisational games (cf. Greenbaum \& Kyng (eds.), 1991), and a broad range of more traditional user testing techniques in order to ensure the presence of conditions for development and transparency, in the interface. For example; Frederiksen et al. (in press) has showed that inconsistency in scrolling direction on word-processors and scrolling direction in molecule-modelling tools yields the most transparent interaction for chemistry students; questioning the universality of the common belief that the universal direction of scroll bars should be "up moves the window up".

The challenge in designing for transparency is to ensure that the user interface provides the conditions for creating a zone of proximal development of operations. That is: designing the interface to mediate the initiation of and learning through exploratory actions, subsumed under the overall use-activity; that the interface enables the user to look ahead of the zone of actual development, and to explore conditions for obtaining different new results; and through this exploration support the development of new ways of realising the object of the activity, without entering another separately motivated activity.

While it is impossible to give general guidelines concerning specific features of "transparent interfaces"; it is possible to elaborate on the three issues concerning the development of transparent interaction stated above, in order to yield a more design oriented understanding of the conditions for development of transparency. That is, supporting development in use, ensuring a certain degree of initial familiarity and setting conditions for the formation of new operations.

\subsection{Development in use}

The first issue deals with the necessity to support the development of the use-activity within the use-activity. In general, we cannot completely avoid separately motivated learning activities like reading introductory documentation, attending guided tours, etc. If users do not know what a mouse is and how to operate it, they will not be able to do anything with, e.g. a Macintosh computer. In such situations the only solution is a more explicit socially mediated way of learning; via personal guidance, or by written advises on the screen or in a manual. With respect to transparent interaction the interesting issue, however, is how support for development can be an integrated part of day to day use, when a use situation is established.

The main problem with UNIX and Emacs is that although the possibilities for transparent interaction exist for programmers the actualisation of these possibilities is difficult due to the absence of support for learning within the use activity. To learn 
something about the systems it is necessary to consult manuals or colleagues, which possess more knowledge about the system, thus separating learning from doing. The use of on-line help systems, especially context sensitive help-systems, can be seen as a way of supporting development in use. However, an on-line help system can also drag the user away from the use-activity by being too difficult to use. Furthermore, the entire concept of on-line help is based on the idea of separating learning from doing by providing users with external descriptions instead of supporting development directly by features of the interface.

\subsection{Initial familiarity}

The second issue deals with the user's ability to reflect the potential mastering of the computer - to look into a zone of proximal development. The interface must provide users with an idea or initial familiarity of what they might be able to do in near future, and thereby encourage them to initiate exploratory learning actions. The concept of affordance (Gibson 1979) captures this at a very low level of interaction; the properties of an object give the user an idea of its potential use. On a more overall level the use of metaphors can give the user an idea of potential use of the artefact by providing an analogy between something known and concrete, and something unknown and abstract. In the same manner, consistency between applications, e.g. the organisation of menus on the Macintosh, supports the transfer of skills from one application into initial familiarity with another.

Because learning (as opposed to mere adapting) requires conscious reflection of the conditions for the future operation, an exploratory learning action will interrupt the transparent flow of operations. This issue of providing users with an initial familiarity, therefore implies a trade-off between inviting (or even forcing) them to initiate learning; and allowing them to continue the accomplishment of their jobs. Therefore we propose that the emphasis must be on the clues built into the interface; this could be called graceful tutoring.

The initial familiarity encouraging exploration must not inhibit the ongoing use of the artefact but, on the other hand, the interface must exhibit the possibilities of the artefact in order to give users ideas of its future use. The way this trade-off has to be solved cannot be answered in general. It very much depends on who is going to use it, their use activity, the need the artefact tries to solve, the motive for the users to use it, the context of use, etc. A simple issue as whether it is sufficient to present the functionality of a window based application in pull down menus or whether it should be represented as buttons and icons, depends on the way the application is going to be used. If it is going to be used often, having buttons and icons all over the screen might be very annoying, especially if the space could be used for another application supporting the current activity. But, on the other hand, hiding functionality away in a pull down menu might prevent the casual user from learning about the different possibilities of the application. Thus, it is necessary to know how the artefact is going to be used in order to establish exactly how the interface should invite users to exploration.

\subsection{The formation of new operations}

The third issue deals with the formation of operations. Once exploratory actions have been initiated, the conditions provided by the interface determine how an operation is 
established. Thus the interface should set conditions that support the formation and mastering of actions that are generalised and brief. The essential aspect of the formation of new actions is the development of the orienting basis for the actions. Thus, designers should consider how the creation of a generalised orienting basis can be supported.

UNIX has highly general commands and a basic structure (pipe-lining etc.) that supports this generality, thus the operating system yields a possibility for highly generalised actions of using the commands. Furthermore, it has the potentials for abbreviated actions. UNIX does not, however, support the development of a generalised orienting basis for the actions of execution of these commands. Hence the general and brief usage of UNIX is seldom achieved through use.

Feedback from the artefact about results obtained in the course of mastering an exploratory action determines how the user learns about the artefact. The command based interaction of UNIX only supports trial-and-error learning because the feedback on commands consists only of prompting for a new command, indicating that the system has done something, or displaying uninformative error messages like "Command not found". A more analytical learning of UNIX requires that users get to understand the structure of the operating system - the file system, pipes etc. The only obvious way for users to obtain this knowledge is to enter a separately motivated and detached activity of learning the basic concepts of the operating system.

To support the development of transparent interaction the designer must ensure that feedback from the artefact provides users with a general understanding of the artefact and its use. From the feedback and other cues users must be able to achieve an understanding of the full range of an action, the underlying assumptions, the different results that can be obtained, and how it can be performed in a brief way. This is to learn how the artefact mediates an activity directed toward an object and master the operation necessary for using the artefact in an activity. If the object of the interaction is to handle files and programs in an operating system like UNIX, feedback about the state of the file system (e.g. the directory structure, list of files in a directory etc.) is essential. Emacs supports feedback about matching parenthesis, indentation of nested loops, etc. and therefore mediates a transparent interaction toward the object of writing source code. Mastering an action is the ability to perform it in novel situations with unexpected materials; if users are supplied with sensible feedback and the possibility of reversing actions with unwanted results, the distance between exploring and using the artefact becomes smaller.

The designer supports the development of transparent interaction embedded in use through the lay-out of the artefact and the various kinds of feedback from the artefact. The overall arrangement and behaviour of items on a screen can provide cues to the underlying structure of the application and thereby support the development of transparency in use. How the initial familiarity and the feedback during use should be designed in a specific artefact, depends on the object of the interaction. Therefore, in order to design the way the artefact looks and responds during use we need knowledge about the structure of the activity that the computer artefact is intended to mediate and the object of this activity. Because knowledge about how a new artefact will mediate an activity cannot be obtained separated from the activity the designer needs to apply methods for designing the artefact in use. This calls for prototyping and other participatory design techniques. 


\section{Conclusion}

The starting point of this paper was that HCI research based on information processing psychology is unable to comprehend the conditions for the widely accepted desire to design for transparency. This deficiency was identified as a result of the failure to recognise the dialectical nature of human beings' relations to their surroundings. The conceptual framework of activity theory made it possible to analyse the otherwise vague concept of transparency. This showed that transparency is not a static feature of the interface but an evolving qualitative aspect of the relation between human beings and computer artefacts. Transparency of interaction depends on the learning process that goes on synchronously with the process of use; thus, the important issue for the interface designer is to design the conditions for this development.

We have pointed at the need for a thorough reconsideration of the theoretical foundation for human-computer interaction. We do not reject the established pool of results from mainstream HCI research and practice, but we find that the present lack of a coherent theoretical foundation is preventing the field from taking the next great step forward. We have pointed at activity theory as a possible foundation for HCI. By doing this we do not suggest that activity theory is the only valid theoretical foundation or that it does not have limitations, but we think it is worthwhile to catch up on the growing interest in activity theory in the HCI community (e.g. Bødker 1991, Nardi 1995, Bannon \& Bødker 1991, Bannon \& Kuutti 1993).

The main conclusions with respect to design is that the inseparability of learning and doing should be taken into account by designing a curriculum for development integrated in the computer artefact. Development of transparent interaction can be supported by providing cues and feedback according to the structure of this object. This should be based on analysis of the object of the activity, that the artefact is supposed to mediate. The aspects of transparent interaction discussed can serve as points to consider during the design process. We hope that our theoretical contribution can be a source of inspiration for designers.

\section{Acknowledgements}

We want to thank Annette Aboulafia, Susanne Bødker, Tania Funston, Kim Halskov Madsen, Niels Jacobsen and the anonymous reviewers for comments on this paper.

\section{References}

Anderson, John R. (1983), The Architecture of Cognition, Cambridge MA: Harvard University Press.

Bannon, L. (1986) Helping Users Help Each Other, in Norman \& Draper (eds.), User Centered System Design, San Diego.

Bannon, L. \& Bødker, S. (1991) Beyond the Interface: Encountering Artifacts in Use, in Carroll, John M. (ed.) Designing Interaction.

Bannon, L. J. \& Kuutti, K. (1993) Searching for Unity among Diversity: Exploring the "Interface" Concept, in Proceedings of INTERCHI 1993. 
Bol, E. (1984) On the Development of Learning Activity. In Hedegaard, M., Hakkarainen, P. \& Engeström, Y. (Eds.) Learning and Teaching on a Scientific Basis, Aarhus University, Institute of Psychology.

Bødker, S. (1991) Through the interface: a human activity approach to user interface design, Hillsdale, N.J.

Card, S. K.; Moran, T. P. \& Newell, A. (1983): The Psychology of Human-Computer Interaction, Hillsdale NJ.

Feyerabend, P. (1975), Against Method, London.

Frederiksen, N., Grudin, J. \& Laursen, B. (In press) Inseparability of Design and Use: An Experimental Study of Design Consistency. To appear in Proceedings of the Computer In Context Conference, Aarhus Denmark, August 1995.

Gal'perin, P. Y (1969) Stages in the Development of Mental Acts, in Cole \& Maltzman (eds.) A Handbook of Contemporary Soviet Psychology, New York: Basic Books.

Gibson, J. (1979) The Ecological Approach to Visual Perception, Houghton Mifflin, Boston.

Greenbaum, J. \& Kyng, M. (eds.) (1991), Design at Work, Hillsdale: LEA.

Habermas, J. (1972) Knowledge and Human Interests, Boston: Beacon Press.

Hutchins, E.L.; Hollan, J. D. \& Norman D.A. (1986), Direct manipulation interfaces. in Norman \& Draper (eds.), User Centered System Design., San Diego.

Laurel, B. (1986), Interface as Mimesis, in Norman \& Draper (eds.),User Centered System Design., San Diego.

Leontjev, A. N. (1978) Activity, consciousness, and personality, Engelwood Cliffs NJ: Prentice Hall.

Leontjev, A. N. (1981) Problems of the development of the mind, Moscow: Progress

Nardi, B. (ed.) (1995) Context and Consciousness: Activity Theory and Human Computer Interaction, Cambridge: MIT Press.

Newell, A. \& Card, S. K. (1985): The Prospects for Psychological Science in HumanComputer Interaction, in Human Computer Interaction 1985 vol. 1, pp. 209-242.

Newell, Allen (1990), Unified Theories of Cognition, Cambridge Ma.

Norman, D. A. (1981) The Trouble with UNIX, in Datamation vol. 27 no 7.

Norman, D. A. \& Draper, S. (eds.) (1986), User Centered System Design., San Diego.

Shneiderman, B. (1992) Designing the user interface, Reading MA.

Snoddy, G. S. (1926), Learning and Stability, in The Journal of Applied Psychology vol. 10 pp. 1-36.

Strong, G. W. (ed.) (1994), New Directions in Human-Computer Interaction Education, Research, and Practice, Drexel.

Vygotsky, L.(1978) Mind in society: The development of higher mental processes, Cambridge, MA.

Winograd, T. \& Flores, F. (1986), Understanding Computers and Cognition -- a new foundation for design, Norwood, N.J: Ablex. 